\title{
Radiotherapy in women with epithelial ovarian cancer: historical role, current advances, and indications
}

\author{
Christian Haydeé Flores-Balcázar ${ }^{1,2}$, Dulce María Urías-Arce ${ }^{1}$ \\ ${ }^{1}$ Radiotherapy and Medical Physics Service, Instituto Nacional de Ciencias Médicas y Nutrición Salvador Zubirán, México City, México; \\ ${ }^{2}$ Radiotherapy Service, Division of Breast and Gynecological Tumors, Instituto Nacional de Cancerología, México City, México \\ Contributions: (I) Conception and design: CH Flores-Balcázar; (II) Administrative support: DM Urías-Arce; (III) Provision of study materials or \\ patients: All authors; (IV) Collection and assembly of data: DM Urías-Arce; (V) Data analysis and interpretation: All authors; (VI) Manuscript \\ writing: All authors; (VII) Final approval of manuscript: All authors. \\ Correspondence to: Christian Haydeé Flores-Balcázar. Radiotherapy and Medical Physics Service, Instituto Nacional de Ciencias Médicas y Nutrición \\ Salvador Zubirán, 15 Vasco de Quiroga Ave., Belisario Domínguez, Sección XVI, Tlalpan,14080, México City, México. Email: chrishaydee@hotmail.com.
}

\begin{abstract}
Epithelial ovarian cancer (OC) is known to be a neoplasm responsive to radiotherapy (RT). Nevertheless, the role of RT in the management of this disease represent a topic of controversy, and the indications for its use are not fully established. Initial studies suggested that the addition of RT in the form of intraperitoneal (IP) radioisotopes was useful. Indications for this treatment were peritoneal cytology with tumor cells, peritoneal implants, and capsule rupture. The instillation of radioisotopes was contraindicated when macroscopic residual disease was present. Pelvic RT was used after surgery in patients with an absence of gross residual disease. Early studies established the inadequacy of this technique and the need for treating the whole abdomen. Whole abdominal irradiation (WAI) was a therapeutic tool used in the prechemotherapy era to eradicate large amounts of microscopic peritoneal disease. Ideal candidates for WAI were stage I patients with grade 2 or 3 tumors; stage II patients with grade 1 or 2 tumors and residual disease, and stage III, grade 1 patients with $<2 \mathrm{~cm}$ residual disease. The disadvantages of WAI were the dose-limiting toxicities, which were predominantly acute hematologic and late gastrointestinal. The era of aggressive debulking and platinum agents made WAI fall out of favor as a treatment of OC. Selective approaches with highly conformal radiotherapy (CRT) have been used in case of limited recurrent or unresectable disease with the potential for long-term disease control. Currently, the role of RT in OC applies for patients with recurrent oligometastatic or oligoprogressive disease and in the palliative setting for symptom control. We performed a nonsystematic review and included data from both retrospective and prospective studies focusing on the use of RT for OC and its biological rationale. Furthermore, ongoing trials on this issue are reported.
\end{abstract}

Keywords: Immunotherapy ovarian cancer (immunotherapy OC); radiosensitizers; radiotherapy (RT); stereotactic body radiotherapy (SBRT)

Submitted Jan 07, 2020. Accepted for publication Jul 06, 2020.

doi: $10.21037 /$ cco-20-10

View this article at: http://dx.doi.org/10.21037/cco-20-10

\section{Introduction}

Ovarian cancer (OC) is one of the most common malignancies around the globe, with 295,414 newly diagnosed cases and 184,799 deaths registered in 2018, according to Globocan (1). Because of a silent spillage OC is mainly diagnosed as an advanced disease (the most common stage at presentation); being the most lethal gynecological malignancy $(2,3)$. The World Health Organization histological classification for ovarian tumors separates ovarian neoplasms according to the probability of origin: Surface epithelial (65\%), germ cell (15\%), sex cord-stromal $(10 \%)$, metastatic $(5 \%)$ and miscellaneous $(5 \%)$. Surface epithelial tumors are further classified by cell type (serous, 
mucinous, endometrioid) and atypia [benign, borderline (atypical proliferation, low malignant potential)] or malignant (4). The hallmark of OC biology is intraperitoneal (IP) dissemination. Early OC stages may present with an ovarian tumor in nearly $30 \%$ of cancer cases (5). Once the tumor mass overpasses the ovarian surface, tumor cells disseminate through the IP fluid and transports malignant cells in a diffuse peritoneal distribution (5).

Once OC is diagnosed, treatment is based on the stage of disease, age, performance status, life expectancy, and comorbidities. Staging is performed with hysterectomy, bilateral salpingo-oophorectomy, pelvic and paraaortic lymph node dissections, omentectomy, peritoneal washings, and peritoneal biopsies (6). Therapeutic options include surgery, chemotherapy with novel targeting molecular therapies, radiotherapy (RT), or a combination of these modalities (7). Treatment with chemotherapy depends on surgical staging findings, the grade and the histology of disease. Complete surgically staged patients with grade I tumors can be observed with no additional treatment, whereas for patients with grade II and grade III or advanced ovarian carcinoma a comprehensive debulking surgery is the mainstay of management followed by adjuvant platinum/ taxane-based chemotherapy for 3 to 6 cycles $(5,8)$.

The role of RT in the management of OC continues to represent a topic of controversy, and the indications for its use are not fully established. Thus, to assess the use of RT in $\mathrm{OC}$, we conducted a literature search of all publications written in English language through the PubMed/Medline central database at the National Center for Biotechnology Information (NCBI) website (9) searching by the terms "ovarian cancer", "radiation therapy", "radiotherapy", "whole abdominal irradiation", "intraperitoneal radionuclides", and "stereotactic body radiotherapy". The last search date was December 16, 2019.

\section{Role of adjuvant radiation therapy for early-stage OC}

Epithelial OC is known to be a radiosensitive tumor. Initial studies suggested that the addition of RT in the form of IP radioisotopes and whole abdominal irradiation (WAI) to chemotherapy for subsets of patients was useful.

\section{IP instillation of radioisotopes}

Because of the risk of IP dissemination and before the platinum-based chemotherapy era, the benefit of adjuvant peritoneal administration of radionuclides, such as gold $\left({ }^{198} \mathrm{Au}\right)$ and chromic phosphate $\left({ }^{32} \mathrm{P}\right)$, was evaluated in a group of studies (10-15). Indications for this treatment were peritoneal cytology with tumor cells, peritoneal implants, and capsule rupture. The instillation of radioisotopes was contraindicated when macroscopic residual disease was present. The first studies were performed using colloidal gold, because of its short half-life (2.697 days) and decay properties. The radiation was mainly negative beta decay with a mean energy of $0.316 \mathrm{MeV}$ and a maximum energy of $0.96 \mathrm{MeV}$. The gamma decay energy was $411 \mathrm{keV}$. The therapeutic effect was due to the short-range beta radiation according to physical properties (its $3.8 \mathrm{~mm}$ penetration range in water/soft tissue destroyed tumors without nearby healthy tissue being irradiated). The handling of ${ }^{198} \mathrm{Au}$ was complicated because of higher risks of exposure for the staff and the need to separate the patients from one another (16). In addition, the physical properties of ${ }^{32} \mathrm{P}$ were more convenient because of higher beta energy (mean energy of $0.69 \mathrm{MeV}$ ) with a maximum energy of $1.709 \mathrm{MeV}$, and a maximum penetration in tissue of $7.6 \mathrm{~mm}$, longer halflife than ${ }^{198} \mathrm{Au}$ (14.29 days), easier handling, lack of gamma radiation, and a relatively low complication rate (17).

Three trials evaluated IP ${ }^{32} \mathrm{P}$ or adjuvant chemotherapy in patients with high-risk, early-stage tumors. IP ${ }^{32} \mathrm{P}$ resulted in increased bowel toxicity and not superior than chemotherapy in survival outcomes (Table 1) (18-20). The use of ${ }^{32} \mathrm{P}$ for early-stages of $\mathrm{OC}$ was abandoned due to the new platinum agents. A better understanding of the physiopathology of OC led to a better classification of earlystage patients in whom close observation could be feasible instead adjuvant therapy (21). For patients with advancedstage OC, ${ }^{32} \mathrm{P}$ in combination with WAI was attempted but found to be excessively toxic with no difference in relapse rates or survival (22-24). At present, IP instillation of radioisotopes is no longer a therapeutic strategy for OC.

\section{Adjuvant pelvic RT}

Before WAI, pelvic RT was used after surgery in patients with an absence of gross residual disease (25). Early studies established the inadequacy of this technique and the need for treating the whole abdomen. Patients with microscopic residual disease after initial debulking surgery experienced 10-year disease-free survival (DFS) rates of $42 \%$ to $62 \%$ in two randomized trials evaluating pelvic RT $(26,27)$. A trial from Dembo et al., randomized 147 women with OC in stages I-III to either abdominopelvic 
Table 1 Prospective studies of adjuvant ${ }^{32} \mathrm{P}$ IP instillation in high-risk ovarian carcinoma

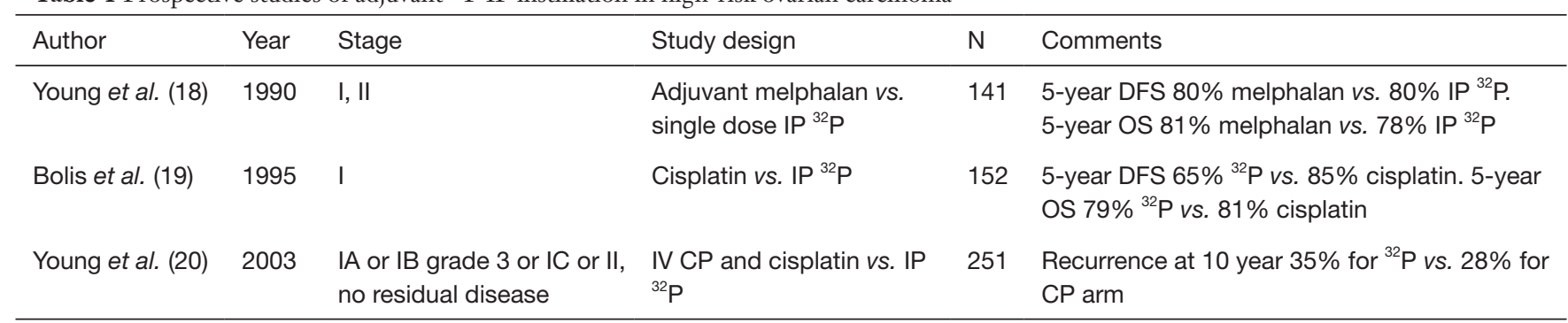

IP, intraperitoneal; DFS, disease-free survival; OS, overall survival; CP, cyclophosphamide.

RT without chemotherapy, pelvic RT alone or pelvic RT followed by adjuvant daily chlorambucil therapy. For analyzing survival characteristics patients were divided in two groups according to complete or incomplete debulking surgery. The 5 -year actuarial survival rate after complete debulking surgery was $81 \%$ among patients treated with abdominopelvic RT and $51 \%$ in patients treated with pelvic RT plus chlorambucil $(\mathrm{P}=0.019)$. This benefit appeared to be independent of stage. Poor prognosis for patients with incomplete debulking surgery was not different for any of the tested therapies. Serious gastrointestinal toxicity was uncommon in the abdominopelvic RT arm with one patient requiring bowel surgery. The pelvic RT only and pelvic RT and chlorambucil arms had eight cases of gastrointestinal toxicity (abdominal cramps and rectal bleeding) (28). A trial from Hreshchyshyn et al. randomized 86 stage-I OC patients to pelvic RT, melphalan, or no additional treatment. A survival difference between no additional treatment and pelvic RT could not be demonstrated but a benefit for melphalan was obtained (29). Sell et al. published a study of 118 patients randomized to WAI or pelvic RT with cyclophosphamide (CP). There was no difference between the regimens with respect to recurrence-free survival (RFS) (55\%) and 4-year overall survival (OS) (63\%). At routine second-look laparotomy (SLL), 16\% of patients without clinical detectable tumor showed recurrence (30). These trials validated RT as an effective adjuvant modality in patients with OC. They also stablished that WAI is better than pelvic RT alone or in combination with chemotherapeutic agents for patients with microscopic or no residual disease.

\section{Adjuvant WAI}

Adjuvant WAI was a therapeutic tool used in the prechemotherapy era to eradicate large amounts of microscopic peritoneal disease. Ideal candidates for WAI were stage I patients with grade 2 or 3 tumors; stage II patients with grade 1 or 2 tumors and residual disease, and stage III, grade 1 patients with $<2 \mathrm{~cm}$ residual disease (26). Its advantage in comparison to ${ }^{32} \mathrm{P}$ and pelvic $\mathrm{RT}$ was the ability to deliver a homogeneous radiation dose to all areas of the abdomen and pelvis encompassing pelvic and paraaortic lymph nodes. The disadvantages of WAI were the dose-limiting toxicities, which were predominantly acute hematologic and late gastrointestinal. The total dose in WAI technique was limited to 25-30 Gy, owing to the radiation tolerance of organs within the field (31). Vergote et al. randomized 347 patients, stages I to III (no macroscopic residual disease), to cisplatin $\left(50 \mathrm{mg} / \mathrm{m}^{2}\right)$ every 3 weeks for six cycles or ${ }^{32} \mathrm{P}$. Patients with intraabdominal adhesions initially randomized to IP administration of ${ }^{32} \mathrm{P}$ received WAI followed by pelvic RT instead. The 5 -year OS for patients with stage I disease was $82 \%, 94 \%$, and $79 \%$ for the ${ }^{32} \mathrm{P}$ arm, WAI and cisplatin arms respectively. Bowel obstruction was frequent in patients treated with ${ }^{32} \mathrm{P}$ (9\%) and WAI (21\%) when compared with to cisplatin arm (2\%) (32). Because no survival benefit was shown with RT, cisplatin was recommended as the standard treatment for this subset of patients (Table 2) (33-35).

\section{Role of consolidative radiation therapy for advanced-stage ovarian carcinoma}

The role of consolidative RT in OC besides surgery and chemotherapy has been controversial. Several publications addressed the benefit of RT in this scenario.

\section{Consolidative WAI}

Consolidative WAI was used after SLL in patients with minimal residual disease and without prior abdominopelvic RT to eradicate subclinical residual disease in women 
Table 2 Prospective studies of adjuvant WAI in ovarian carcinoma

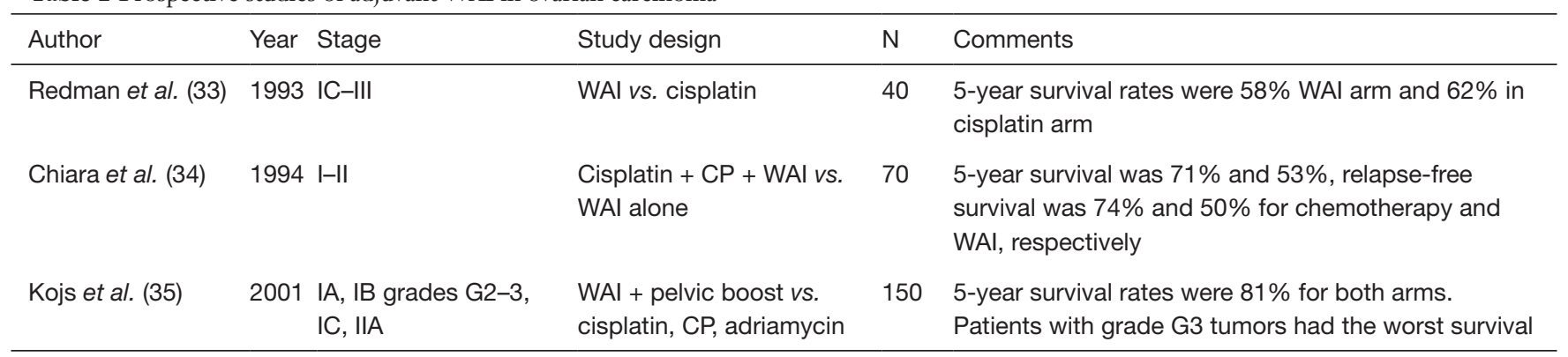

WAI, whole abdominal irradiation; CP, cyclophosphamide.

Table 3 Prospective studies of consolidative WAI in ovarian carcinoma

\begin{tabular}{|c|c|c|c|c|c|}
\hline Author & Year & Stage & Study design & $\mathrm{N}$ & Comments \\
\hline Lambert et al. (41) & 1993 & IIB-IV & Carboplatin vs. WAI & 254 & $\begin{array}{l}\text { Median survival } 25 \text { months for both groups. No } \\
\text { statistical difference in OS or DFS between groups }\end{array}$ \\
\hline Pickel et al. (43) & 1999 & IC-IV & WAI vs. observation & 64 & $\begin{array}{l}5 \text {-year OS rate of } 59 \% \text { in WAI arm and } 33 \% \text { for } \\
\text { observation arm }\end{array}$ \\
\hline $\begin{array}{l}\text { Sorbe and Swedish- } \\
\text { Norwegian Ovarian } \\
\text { Cancer Study Group (44) }\end{array}$ & 2003 & $\begin{array}{l}\text { III without residual } \\
\text { disease. Macroscopic } \\
\text { disease }\end{array}$ & $\begin{array}{l}\text { WAI vs. chemotherapy } \\
\text { vs. no further therapy. } \\
\text { WAI vs. chemotherapy }\end{array}$ & 172 & $\begin{array}{l}5 \text {-year PFS rate } 56 \% \text { WAl arm vs. } 36 \% \\
\text { chemotherapy arm vs. } 35 \% \text { no treatment group. No } \\
\text { significant differences in survival between arms. Late } \\
\text { severe intestinal radiation reactions in } 10 \%\end{array}$ \\
\hline
\end{tabular}

WAI, whole abdominal irradiation; SLL, second-look laparotomy; OS, overall survival; DFS, disease-free survival; PFS, progression-free survival.

who remained at high risk for relapse following surgical cytoreduction and adjuvant chemotherapy. Published data demonstrated 3-year progression-free survival (PFS) rates of $50 \%$ to $67 \%(36,37)$ and 5 -year OS rates of $40 \%$ to $66 \%$ $(38,39)$. A few early, prospective randomized trials evaluated the role of consolidative WAI compared to extended chemotherapy in patients with advanced-stage disease (stage III/IV) after initial surgical cytoreduction, adjuvant chemotherapy, and SLL. In all of these trials, DFS and OS rates were not found to be significantly different between WAI and chemotherapy (Table 3) (40-44).

\section{Consolidative IP ${ }^{32} P$}

Randomized data evaluating IP ${ }^{32} \mathrm{P}$ as consolidative treatment following SLL are limited. Vergote et al. randomized 50 patients with negative second-look findings to receive ${ }^{32} \mathrm{P}$ or no treatment. PFS was $95 \%$ in the ${ }^{32} \mathrm{P}$ arm vs. $82 \%$ for the observation arm. ${ }^{32} \mathrm{P}$ therapy was associated with bowel complications (45). A trial of the Gynecologic Oncology Group randomized 202 patients with a negative SLL to receive ${ }^{32} \mathrm{P}$ or no additional therapy. With a median follow-up of 63 months in living patients, the 5-year RFS rate was $42 \%$ and $36 \%$ for the $\mathrm{IP}^{32} \mathrm{P}$ and no further therapy groups, respectively; the difference was not statistically significant, $61 \%$ of stage III OC patients had tumor recurrence within 5 years of negative SLL (24). These data did not support the use of ${ }^{32} \mathrm{P}$ for consolidative treatment in OC.

\section{Role of RT for clear-cell and mucinous histologies}

Clear-cell carcinoma (CCC) of the ovary is a different histologic subtype of OC that is associated with worse 
outcomes when compared with other subtypes of epithelial OC of a similar stage. Given the relatively chemotherapyresistant nature of ovarian CCC to standard therapy with paclitaxel and carboplatin, different treatment strategies have been evaluated. Because of the modest response rates seen with platinum-based chemotherapy, RT has also been evaluated as a component of treatment in both the first-line and the recurrent setting (46).

Two studies have evaluated surgery plus either chemotherapy and RT or RT alone for upfront treatment of CCC. In a retrospective cohort of 241 patients with stage I and II ovarian CCC treated with surgery followed by platinum/taxane chemotherapy with or without RT, patients with stage IC to II disease had an improvement in DFS that favored RT [risk ratio, $0.54 ; 95 \%$ confidence interval (CI), 0.33-0.95; $\mathrm{P}=0.02]$, and a $20 \%$ increase in DFS at 5 years (47). A second cohort of 28 patients with stage IC to III ovarian CCC treated with surgery followed by either platinum/taxane chemotherapy or WAI showed an improvement in both 5-year OS and DFS in patients who received surgery plus RT (OS: $81.8 \%$ vs. $33.3 \%, \mathrm{P}=0.031$; DFS: $81.2 \%$ vs. $25 \%, \mathrm{P}=0.006$ ) (13). RT has also shown to improve outcomes when used to treat advanced or recurrent ovarian CCC. In a retrospective cohort of 158 patients with stage I to IV ovarian CCC that included recurrent disease, 17 patients received RT either alone or with chemotherapy. In this cohort, $70.6 \%$ of the patients had a treatment benefit when adjuvant treatment included radiation compared with only $27.3 \%$ of the patients treated with chemotherapy alone (48). In another more recent cohort, definitive RT was used to treat 102 women with recurrent OC and included all epithelial histologic subtypes. Subgroup analysis showed that patients with CCC had higher 5-year OS and PFS rates than patients with other histologies (OS: $88 \%$ vs. $37 \%, \mathrm{P}=0.05$; PFS: $75 \%$ vs. $20 \%, \mathrm{P}=0.01$ ) (49). Similar results were also seen for patients with stage I or II OC of endometrioid, clear cell, and mucinous subtypes in a population-based study, which reported a $40 \%$ reduction in disease-specific mortality and $43 \%$ reduction in overall mortality with the addition of consolidative RT (14).

Mucinous ovarian cancer (MOC) is a rare subtype of OC. It has a distinct natural history, molecular profile, chemosensitivity, and prognosis in comparison to other histologies of OC. Recently, a study published by Patel et al., analyzed incidence, survival, and treatments from the Surveillance, Epidemiology, and End Results (SEER) Program for clear cell, mucinous, and endometrioid histologies of the ovary receiving adjuvant RT. For stage I and II patients OS was higher in individuals who did not receive RT at 5 years $(76 \%$ vs. $70 \%)$ and 10 years $(65 \%$ vs. $49 \%, \mathrm{P}=0.036)$ whereas in stage III patients there was an improvement with RT in OS at 5 years $(53 \%$ vs. $36 \%)$ and 10 years $(45 \%$ vs. $26 \%$, $\mathrm{P}=0.052$ ) suggesting $\mathrm{RT}$ could be useful in this setting (50).

\section{Reasons WAI is no longer a standard treatment in OC}

The toxicity of WAI technique is not negligible. During treatment, patient complaints where about diarrhea, fatigue, nausea, and hematologic effects (mainly thrombocytopenia). More concerning, however, used to be long-term toxicities (51). A study published by Thomas et al. analyzed 1,098 patients from 10 prospective series with WAI as OC treatment. Fatigue was the most common complaint. Approximately $75 \%$ of patients had mild diarrhea, $67 \%$ were nauseous and $15 \%$ of patients had bloating. Basal pneumonitis or fibrosis was evident in chest radiographs in up to $20 \%$ of patients. Transient elevation of alkaline phosphatase levels occurred in $50 \%$ of patients. The necessity for bowel surgery for treatment complications was $5.6 \%$. Four patients died as a result of bowel damage (52). The era of aggressive debulking and platinum agents made WAI fall out of favor as a treatment of OC (51).

\section{Current scenarios for RT in OC}

\section{Salvage RT for recurrent disease}

Abdominopelvic relapse is the main pattern of failure in OC patients treated with definitive and adjuvant therapy. The response rates to second-line chemotherapy in patients with relapsed OC are poor. Salvage WAI (30-35 Gy, followed by a pelvic boost), has been employed in an effort to improve outcomes (53). Selective approaches with highly conformal radiotherapy (CRT) have been used in case of limited recurrent disease (nodal or extranodal non-debulkable disease) with no disease dissemination, a subset of these patients may have oligometastatic or oligoprogressive disease that may beneficiate from RT instead of surgery with the potential for long-term disease control (Table 4) (54-58).

\section{Symptom control in the palliative setting}

Palliative RT in OC is effective in patients with symptomatic localized disease. For patients with vaginal bleeding, pain resulting from retroperitoneal mases, or 
Table 4 Studies of RT in recurrent ovarian carcinoma

\begin{tabular}{|c|c|c|c|c|c|}
\hline Author & Year & Stage & Study design & $\mathrm{N}$ & Comments \\
\hline Komura et al. (57) & 2019 & $\begin{array}{l}\text { Nodal or extranodal } \\
\text { recurrence }\end{array}$ & Retrospective 3D-CRT & 24 & $\begin{array}{l}\text { In-field overall response of } 58.3 \% \text {, median } \\
\text { regression was } 40.2 \% \text {. The } 1 \text {-year survival } \\
\text { and local PFS rates after RT were } 66.7 \% \text { and } \\
45.8 \% \text {, respectively }\end{array}$ \\
\hline
\end{tabular}

IFRT, involved-field radiotherapy; LRFS, local recurrence-free survival; DFS, disease-free survival; 3D-CRT, three-dimensional conformal radiotherapy; LC, local control; BED, biological equivalent dose; CRR, complete response rate; PFS, progression-free survival; OS, overall survival; IMRT, intensity-modulated radiation therapy.

metastatic disease (e.g., brain metastases, voluminous or painful nodes), RT can induce tumor regression and provide symptom relief. Bansal et al. (59) analyzed abdominal pain and vaginal bleeding control in a group of 23 patients with metastatic OC treated with pelvic RT. After finishing RT, abdominal pain was controlled in $88.2 \%$ and vaginal bleeding in $80 \%$ with doses ranging from 46-50 Gy. Jiang et al. (60) published a study of 64 patients with symptomatic OC recurrence who received RT for pain, bleeding, and obstruction. Overall response rates were higher for bleeding control in $93 \%$ and for pain control in $87 \%$ than for obstruction in $62 \%$. Patients treated for pain at nonbony sites had higher pain control (96\%) compared with those treated at bony sites $(75 \%)$. When delivered locally to symptomatic sites, RT appears to be of significant and durable benefit and should be considered for palliative purposes in select patients with symptomatic relapses, particularly in those who are refractory to chemotherapy.

\section{Feasibility of modern radiation techniques in ovarian carcinoma}

\section{Intraoperative $R T$}

Several studies suggested that intraoperative radiation therapy (IORT) as part of salvage surgery for locally recurrent gynecologic cancers, including OC, may improve local-regional control and OS. However, a few institutions have reported their experiences with IORT for recurrent OC with 5 -year OS of $22 \%$ with a median survival of 26 months from the time of IORT (61). Barney et al. reported a series on 20 women with recurrent $\mathrm{OC}$ who were treated with IORT to the pelvis, para-aortic or inguinal lymph nodes. Eighty percent of women also received perioperative external beam RT. Five-year locoregional control was 59\%, with 14 and 30 months DFS and OS, respectively (62). Given the propensity of OC to recur in the abdomen, IORT may be suitable for patients with isolated recurrences in the retroperitoneum, in patients with isolated pelvic disease after resection and certain histologic subtypes that may be less sensitive to conventional chemotherapy (63).

\section{Intensity modulated-WAI}

The use of intensity-modulated radiation therapy (IMRT) to deliver WAI has been proposed as a means of reducing the radiation dose to the bone marrow and kidneys to decrease the incidence of myelotoxicity and renal 

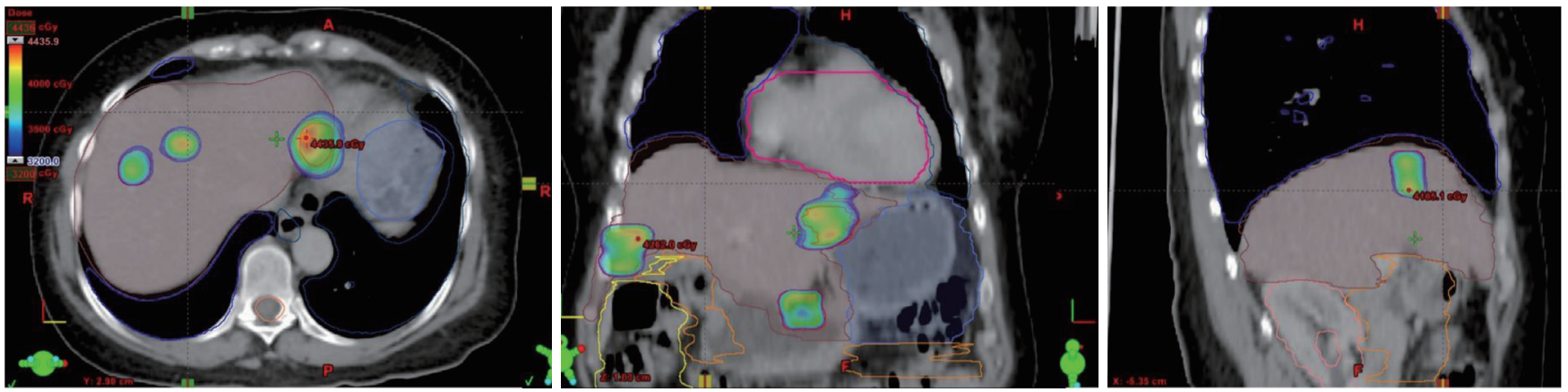

Figure 1 SBRT plan for the treatment of liver metastases in a woman with recurrent MOC is shown. Treatment planning with axial, coronal, and sagittal views is depicted. The PTV is color washed in green, heart is pink, the kidneys are orange and cyan, liver is brown, stomach is blue, and the bowel is orange. The prescription dose was 34 Gy in five fractions. SBRT, stereotactic body radiotherapy; MOC, mucinous ovarian cancer; PTV, planning target volume.

damage. Dosimetric analysis has demonstrated improved planning target volume coverage and significant dose reductions to bones with equivalent kidney-sparing using dynamic multileaf collimator IMRT when compared with conventional fields (64). Recently, the dosimetric advantages of WAI-IMRT and the clinical benefit (CB) of the technique were tested in a prospective phase II study from Arians et al. (65). Twenty patients with optimally cytoreduced stage III OC with complete remission after chemotherapy were treated with WAI-IMRT as consolidation therapy. A total dose of 30 Gy in 20 fractions of 1.5 Gy was applied to the peritoneal cavity. WAI-IMRT resulted in good coverage of the peritoneal cavity with effective sparing of all organs within the peritoneum. The tolerability rate of WAI-IMRT was $>70 \%$. Side effects mostly consisted of grade 1 or 2 gastrointestinal toxicity with nausea, vomiting or diarrhea with an acceptable quality status of health (65).

\section{Stereotactic body radiotherapy (SBRT)}

SBRT is a highly conformal radiation technique that allows doses greater than 5 Gy per fraction to be delivered during a course of 1 to 5 treatments including ablative doses of radiation; these ablative doses of radiation may be used in cases of few metastases or in those with mixed response to chemotherapy, where consolidation treatment can be delivered to the few nonresponding or progressive sites of disease (Figure 1) (66). This approach has been validated in multiple disease sites (lung, prostate, breast) for oligometastases with $80 \%$ to $90 \%$ local control (LC) rates, an improvement in OS, and small risks of significant acute/ late toxicity (AT/LT) (67). The few dedicated reports on
SBRT for OC are enlisted in Table 5 (68-70).

\section{The future of RT for OC}

\section{RT and poly (ADP-ribose) polymerase (PARP) inbibitors}

PARP inhibitors are oral agents that inhibit PARP enzymes 1,2 , and 3 with clinical efficacy among OC patients. PARP inhibitors were the first United States Food and Drug Administration-approved biological agents for OC based on molecular features of cancer (71). Patients with BRCA1/2mutated or homologous recombination-deficient ovarian tumors can benefit from PARP inhibitors. However, data on safety, tolerability and efficacy in combination with RT are limited (72). Reiss et al. tested low-dose WAI with veliparib in patients with peritoneal carcinomatosis adding a dose escalation in ovarian and fallopian cancer patients. Thirty-two patients were treated. Lymphopenia, anemia, and thrombocytopenia where the most common grade 3 and 4 toxicities. After 45 months of follow-up, median PFS was 3.6 months, and median OS was 9.1 months. In OC patients, OS was longer for platinum-sensitive patients (10.9 months) compared to platinum-resistant patients (5.8 months). When combined with WAI, the maximum tolerated dose was determined to be $250 \mathrm{mg}$ twice daily via oral administration (73).

\section{RT and immunotherapy combination}

Clinical remission for OC is possible, however, $70 \%$ of patients will relapse, with 5-year survival rates of approximately $30 \%$ (74), with a proportion of patients 
Table 5 Studies of SBRT/SABR in oligometastatic ovarian carcinoma

\begin{tabular}{|c|c|c|c|c|}
\hline Author & Year & Study design & $\mathrm{N}$ & Comments \\
\hline Lazzari et al. (69) & 2018 & Retrospective SBRT & $\begin{array}{l}82 \text { patients; } \\
156 \text { lesions }\end{array}$ & $\begin{array}{l}\text { Median systemic treatment-free interval after SBRT: } \\
7.4 \text { months, 2-year local PFS: } 68 \% \text {, PFS: } 18 \% \text {, OS: } 71 \% \text {. }\end{array}$ \\
\hline Macchia et al. (70) & 2020 & $\begin{array}{l}\text { Retrospective, multicenter } \\
\text { study (MITO RT-01) } \\
\text { SBRT/SRS }\end{array}$ & $\begin{array}{l}261 \text { patients; } 449 \\
\text { lesions }\end{array}$ & $\begin{array}{l}\text { CRR: } 65.2 \% \text {, PRR: } 23.8 \% \text {, SD: } 7.4 \% \text {, PD: } 3.6 \% \text {; } 2 \text {-year LC: } \\
\text { 81.9\%, ORR: } 89 \% \text {, CB: } 96.4 \% \text {, AT: } 20.7 \% \text {, LT: } 6.1 \% \text {, 2-year } \\
\text { late TFS: } 95.1 \%\end{array}$ \\
\hline
\end{tabular}

SBRT/SABR, stereotactic body radiotherapy/stereotactic ablative radiotherapy; CRR, complete response rate; PRR, partial response rate; $\mathrm{SD}$, stable disease; PD, progressive disease; LC, local control; ORR, objective response rate; CB, clinical benefit; AT, acute toxicity; LT, late toxicity; TFS, toxicity-free survival.

remaining cancer-free at 10 years less than $15 \%$ (75). Immunotherapy has emerged as a therapeutic option. Immune checkpoint inhibitors have gained an important place in the treatment of several disease cancer types (76). However, OC remains poorly responsive to immunotherapy (77).

Both SBRT and WAI could be considered as partners for immunomodulatory therapies to improve tumor control in specific clinical scenarios; SBRT has emerged as an important intervention for in-situ vaccination, while low-dose WAI could be used as a means to achieve subdiaphragmatic so-called in-field tumor reprogramming in the context of immunotherapy schemes (78).

Luke et al. offered SBRT to 2 to 4 metastases to 79 patients who progressed to standard treatment. Patients with ovarian, endometrial, colorectal, and other cancers received 3 to 5 fractions of 10 Gy. Pembrolizumab was initiated within 7 days after completion of SBRT with a dose of $200 \mathrm{mg}$ every 3 weeks until progression, death, or toxic effects. There were 6 dose-limiting toxicity events. There was 1 complete response; 8 partial responses, and 21 patients with stable disease (SD). Multisite SBRT, followed by pembrolizumab, was well-tolerated with acceptable toxicity (79).

\section{Ongoing trials}

A pilot trial (NCT01989546) of BMN 673, an oral PARP inhibitor, is in progress for patients with advanced solid tumors and deleterious BRCA mutations, including OC patients. A phase I trial (NCT03283943) of durvalumab with focal sensitizing RT in platinum-resistant ovarian, primary peritoneal or fallopian tube epithelial carcinoma is also ongoing. A phase I study (NCT03968406) of talazoparib in combination with RT for locally recurrent gynecologic cancers is currently in progress, as is a phase I (NCT03325634) dose-escalation study to determine the maximum tolerated dose of 3 -fraction SBRT for abdominopelvic recurrences of $\mathrm{OC}$ and uterine papillary serous carcinoma. There is also a phase II trial (NCT03618706) aimed to investigate whether the addition of IFRT improves 2-year PFS in patients with recurrent OC after standard primary treatment.

\section{Conclusions}

In the past, RT was frequently used in the management of patients with OC. It was the mainstay of adjuvant treatment for many years but was replaced by cisplatin almost three decades ago. Nevertheless, it remains a useful strategy in patients with recurrent and refractory disease as it can reach a prolonged DFS. The combination of highly CRT techniques with immunotherapy and new radiosensitizers is a paradigm shift for radiation oncology, as the aim of RT is tumor microenvironment reprogramming and immune modulation in addition to tumor ablation. These RT modalities represent new opportunities in OC treatment, promising to enhance the efficacy of new pharmacologic agents in this disease.

\section{Acknowledgments}

Funding: None.

\section{Footnote}

Provenance and Peer Review: This article was commissioned by the Guest Editor (Heriberto Medina-Franco) for the series "Ovarian Cancer" published in Chinese Clinical 
Oncology. The article was sent for external peer review organized by the Guest Editor and the editorial office.

Conflicts of Interest: Both authors have completed the ICMJE uniform disclosure form (available at http://dx.doi. org/10.21037/cco-20-10). The series "Ovarian Cancer" was commissioned by the editorial office without any funding or sponsorship. The authors have no other conflicts of interest to declare.

Ethical Statement: The authors are accountable for all aspects of the work in ensuring that questions related to the accuracy or integrity of any part of the work are appropriately investigated and resolved.

Open Access Statement: This is an Open Access article distributed in accordance with the Creative Commons Attribution-NonCommercial-NoDerivs 4.0 International License (CC BY-NC-ND 4.0), which permits the noncommercial replication and distribution of the article with the strict proviso that no changes or edits are made and the original work is properly cited (including links to both the formal publication through the relevant DOI and the license). See: https://creativecommons.org/licenses/by-nc-nd/4.0/.

\section{References}

1. World Health Organization. International Agency for Research on Cancer Fact Sheet. 2008. Available online: http://gco.iarc.fr/today/data/factsheets/cancers/25-Ovaryfact-sheet.pdf

2. Jayson GC, Kohn EC, Kitchener HC, et al. Ovarian cancer. Lancet 2014;384:1376-88.

3. Zhang Y, Luo G, Li M, et al. Global patterns and trends in ovarian cancer incidence: age, period and birth cohort analysis. BMC Cancer 2019;19:984.

4. Ehdaivand S. WHO classification of ovarian neoplasms. 2012. Available online: https://www.pathologyoutlines. com/topic/ovarytumorwhoclassif.html

5. Orr B, Edwards RP. Diagnosis and treatment of ovarian cancer. Hematol Oncol Clin North Am 2018;32:943-64.

6. Hoskins PJ. Which is the better surgical strategy for newly diagnosed epithelial ovarian cancer: primary or interval debulking? Curr Opin Oncol 2011;23:501-6.

7. Onda T, Yoshikawa H, Yasugi T, et al. Patients with ovarian carcinoma upstaged to stage III after systematic lymphadenctomy have similar survival to Stage I/II patients and superior survival to other Stage III patients.
Cancer 1998;83:1555-60.

8. National Comprehensive Cancer Network. Clinical practice guidelines in oncology (NCCN Guideline): Epithelial Ovarian Cancer (including Fallopian Tube Cancer and Primary Peritoneal Cancer). (version 3.2019). Available online: http://www.nccn.org/

9. National Center for Biotechnology Information, U.S. National Library of Medicine. PubMed Central website. Available online: http://www.ncbi.nlm.nih.gov/pmc

10. Pezner RD, Stevens KR Jr, Tong D, et al. Limited epithelial carcinoma of the ovary treated with curative intent by the intraperitoneal installation of radiocolloids. Cancer 1978;42:2563-71.

11. Dembo AJ. Epithelial ovarian cancer: the role of radiotherapy. Int J Radiat Oncol Biol Phys 1992;22:835-45.

12. Dinniwell R, Lock M, Pintilie M, et al. Consolidative abdominopelvic radiotherapy after surgery and carboplatin/paclitaxel chemotherapy for epithelial ovarian cancer. Int J Radiat Oncol Biol Phys 2005;62:104-10.

13. Nagai $Y$, Inamine $M$, Hirakawa $M$, et al. Postoperative whole abdominal radiotherapy in clear cell adenocarcinoma of the ovary. Gynecol Oncol 2007;107:469-73.

14. Swenerton KD, Santos JL, Gilks CB, et al. Histotype predicts the curative potential of radiotherapy: The example of ovarian cancers. Ann Oncol 2011;22:341-7.

15. Boye E, Lindegaard MW, Paus E, et al. Wholebody distribution of radioactivity after intraperitoneal administration of 32P colloids. Br J Radiol 1984;57:395-402.

16. Patyánik M, Mayer A, Polgár I. Results of ovary tumor treatment with abdominally administered (198)Au evaluated on the basis of long term follow up. Pathol Oncol Res 2002;8:54-7.

17. Currie JL, Bagne F, Harris C, et al. Radioactive chromic phosphate suspension: Studies on distribution, dose absorption, and effective therapeutic radiation in phantoms, dogs, and patients. Gynecol Oncol 1981;12:193-218.

18. Young RC, Walton LA, Ellenberg SS, et al. Adjuvant therapy in stage I and stage II epithelial ovarian cancer. Results of two prospective randomized trials. N Engl J Med 1990;322:1021-7.

19. Bolis G, Colombo N, Pecorelli S, et al. Adjuvant treatment for early epithelial ovarian cancer: results of two randomised clinical trials comparing cisplatin to no further treatment or chromic phosphate (32P). G.I.C.O.G.: Gruppo Interregionale Collaborativo in Ginecologia Oncologica. Ann Oncol 1995;6:887-93.

20. Young RC, Brady MF, Nieberg RK, et al. Adjuvant 


\section{Page 10 of 12}

treatment for early ovarian cancer: a randomized phase III trial of intraperitoneal 32P or intravenous cyclophosphamide and cisplatin-a gynecologic oncology group study. J Clin Oncol 2003;21:4350-5.

21. McGuire WP. Early ovarian cancer: treat now, later or never? Ann Oncol 1995;6:865-6.

22. Tharp M, Hornback NB. Complications associated with intraperitoneal 32P. Gynecol Oncol 1994;53:170-5.

23. Klaassen D, Starreveld A, Shelly W, et al. External beam pelvic radiotherapy plus intraperitoneal radioactive chronic phosphate in early stage ovarian cancer: a toxic combination. Int J Radiat Oncol Biol Phys 1985;11:1801-4.

24. Varia MA, Stehman FB, Bundy BN, et al. Intraperitoneal radioactive phosphorus (32P) versus observation after negative second-look laparotomy for stage III ovarian carcinoma: a randomized trial of the gynecologic oncology group. J Clin Oncol 2003;21:2849-55.

25. Delclos L, Smith JP. Ovarian cancer, with special regard to types of radiotherapy. Natl Cancer Inst Monogr 1975;42:129-38.

26. Smith JP, Rutledge FN, Delclos L. Postoperative treatment of early cancer of the ovary: a random trial between postoperative irradiation and chemotherapy. Natl Cancer Inst Monogr 1975;42:149-53.

27. Dembo AJ. The role of radiotherapy in ovarian cancer. Bull Cancer 1982;69:275-83.

28. Dembo AJ, Bush RS, Beale FA, et al. Ovarian carcinoma: Improved survival following abdominopelvic irradiation in patients with a completed pelvic operation. Am J Obstet Gynecol 1979;134:793-800.

29. Hreshchyshyn MM, Park RC, Blessing JA, et al. The role of adjuvant therapy in stage I ovarian cancer. Am J Obstet Gynecol 1980;138:139-45.

30. Sell A, Bertelsen K, Andersen JE, et al. Randomized study of whole abdomen irradiation versus pelvic irradiation plus cyclophosphamide in treatment of early ovarian cancer. Gynecol Oncol 1990;37:367-73.

31. Klaassen D, Shelley W, Starreveld A, et al. Early stage ovarian cancer: a randomized clinical trial comparing whole abdominal radiotherapy, melphalan, and intraperitoneal chromic phosphate: a National Cancer Institute of Canada Clinical Trials Group report. J Clin Oncol 1988;6:1254-63.

32. Vergote IB, Vergote-De Vos LN, Abeler VM, et al. Randomized trial comparing cisplatin with radioactive phosphorus or whole-abdomen irradiation as adjuvant treatment of ovarian cancer. Cancer 1992;69:741-9.

33. Redman CWE, Mould J, Warwick J, et al. The west
Flores-Balcázar and Urías-Arce. RT in women with epithelial OC

Midlands epithelial ovarian cancer adjuvant therapy trial. Clin Oncol (R Coll Radiol) 1993;5:1-5.

34. Chiara S, Conte PF, Franzone P, et al. High-risk earlystage ovarian cancer: randomized clinical trial comparing cisplatin plus cyclophosphamide versus whole abdominal radiotherapy. Am J Clin Oncol 1994;17:72-6.

35. Kojs Z, Glinski B, Reinfuss $M$, et al. Résultats d'un essai prospectif randomisé comparant une radiothérapie abdominopelvienne postopératoire et une chimiothérapie postopératoire dans les cancers de l'ovaire précoces. Cancer Radiothér 2001;5:5-11.

36. Thomas L, Pigneux J, Chauvergne J, et al. Evaluation of whole abdominal irradiation in ovarian carcinoma with a four orthogonal fields technique. Int J Radiat Oncol Biol Phys 1994;30:1083-90.

37. Kuten A, Stein M, Steiner M, et al. Whole abdominal irradiation following chemotherapy in advanced ovarian carcinoma. Int J Radiat Oncol Biol Phys 1988;14:273-9.

38. Haie C, Pejovic-Lenfant MH, George $M$, et al. Whole abdominal irradiation following chemotherapy in patients with minimal residual disease after second look surgery in ovarian carcinoma. Int $\mathbf{J}$ Radiat Oncol Biol Phys 1989;17:15-9.

39. Bolis G, Zanaboni F, Vanoli P, et al. The impact of wholeabdomen radiotherapy on survival in advanced ovarian cancer patients with minimal residual disease after chemotherapy. Gynecol Oncol 1990;39:150-4.

40. Lawton F, Luesley D, Blackledge G, et al. A randomized trial comparing whole abdominal radiotherapy with chemotherapy following cisplatinum cytoreduction in epithelial ovarian cancer. West Midlands Ovarian Cancer Group Trial II. Clin Oncol (R Coll Radiol) 1990;2:4-9.

41. Lambert HE, Rustin GJS, Gregory WM, et al. A randomized trial comparing single-agent carboplatin with carboplatin followed by radiotherapy for advanced ovarian cancer: a North Thames Ovary Group Study. J Clin Oncol 1993;11:440-8.

42. Bruzzone M, Repetto L, Chiara S, et al. Chemotherapy versus radiotherapy in the management of ovarian cancer patients with pathological complete response or minimal residual disease at second look. Gynecol Oncol 1990;38:392-5.

43. Pickel H, Lahousen M, Petru E, et al. Consolidation radiotherapy after carboplatin-based chemotherapy in radically operated advanced ovarian cancer. Gynecol Oncol 1999;72:215-9.

44. Sorbe B, Swedish-Norgewian Ovarian Cancer Study Group. Consolidation treatment of advanced (FIGO stage 
III) ovarian carcinoma in complete surgical remission after induction chemotherapy: a randomized, controlled, clinical trial comparing whole abdominal radiotherapy, chemotherapy, and no further treatment. Int J Gynecol Cancer 2003;13:278-86.

45. Vergote IB, Winderen M, De Vos LN, et al. Intraperitoneal radioactive phosphorus therapy in ovarian carcinoma. Analysis of 313 patients treated primarily or at second-look laparotomy. Cancer 1993;71:2250-60.

46. Westhoff GL, Fuh KC, Longacre TA, et al. Radiation therapy for recurrent clear-cell cancer of the ovary. Int J Gynecol Cancer 2016;26:1608-14.

47. Hoskins PJ, Le N, Gilks B, et al. Low-stage ovarian clear cell carcinoma: population-based outcomes in British Columbia, Canada, with evidence for a survival benefit as a result of irradiation. J Clin Oncol 2012;30:1656-62.

48. Al-Barrak J, Santos JL, Tinker A, et al. Exploring palliative treatment outcomes in women with advanced or recurrent ovarian clear cell carcinoma. Gynecol Oncol 2011;122:107-10.

49. Brown AP, Jhingran A, Klopp AH, et al. Involved-field radiation therapy for locoregionally recurrent ovarian cancer. Gynecol Oncol 2013;130:300-5.

50. Patel SC, Frandsen J, Bhatia S, et al. Impact on survival with adjuvant radiotherapy for clear cell, mucinous, and endometriod ovarian cancer: the SEER experience from 2004 to 2011. J Gynecol Oncol 2016;27:e45.

51. Fields EC, McGuire WP, Lin L, et al. Radiation treatment in women with ovarian cancer: past, present, and future. Front Oncol 2017;7:177.

52. Thomas GM, Dembo AJ. Integrating radiation therapy into the management of ovarian cancer. Cancer 1993; 71:1710-8.

53. Sedlacek TV, Spyropoulus P, Cifaldi R, et al. Wholeabdomen radiation therapy as salvage treatment for epithelial ovarian carcinoma. Cancer J Sci Am 1997;3:358-63.

54. Albuquerque K, Patel M, Liotta $M$, et al. Long-term benefit of tumor volume-directed involved field radiation therapy in the management of recurrent ovarian cancer. Int J Gynecol Cancer 2016;26:655-60.

55. Choi N, Chang JH, Kim S, et al. Radiation for persistent or recurrent epithelial ovarian cancer: a need for reassessment. Radiat Oncol J 2017;35:144-52.

56. Chang JS, Kim SW, Kim YJ, et al. Involved-field radiation therapy for recurrent ovarian cancer: results of a multiinstitutional prospective phase II trial. Gynecol Oncol 2018;151:39-45.
57. Komura N, Mabuchi S, Isohashi F, et al. Radiotherapy for isolated recurrent epithelial ovarian cancer: a single institutional experience. J Obstet Gynaecol Res 2019;45:1173-82.

58. Smart A, Chen YH, Cheng T, et al. Salvage radiation therapy for localized recurrent ovarian cancer. Int J Gynecol Cancer 2019;29:916-21.

59. Bansal A, Rai B, Kumar S, et al. Fractionated palliative pelvic radiotherapy as an effective modality in the management of recurrent/refractory epithelial ovarian cancers: an institutional experience. J Obstet Gynaecol India 2017;67:126-32.

60. Jiang G, Balboni T, Taylor A, et al. Palliative radiation therapy for recurrent ovarian cancer: efficacy and predictors of clinical response. Int J Gynecol Cancer 2018;28:43-50.

61. Yap OWS, Kapp DS, Teng NNH, et al. Intraoperative radiation therapy in recurrent ovarian cancer. Int J Radiat Oncol Biol Phys 2005;63:1114-21.

62. Barney BM, Petersen IA, Dowdy SC, et al. Intraoperative electron beam radiotherapy (IOERT) in the management of recurrent ovarian malignancies. Int J Gynecol Cancer 2011;21:1225-31.

63. Backes FJ, Martin DD. Intraoperative radiation therapy (IORT) for gynecologic malignancies. Gynecol Oncol 2015;138:449-56.

64. Hong L, Alektiar K, Chui C, et al. IMRT of large fields: whole-abdomen irradiation. Int J Radiat Oncol Biol Phys 2002;54:278-89.

65. Arians N, Kieser M, Benner L, et al. Adjuvant intensity modulated whole-abdominal radiation therapy for highrisk patients with ovarian cancer (International Federation of Gynecology and Obstetrics Stage III): First results of a prospective Phase 2 study. Int J Radiat Oncol Biol Phys 2017;99:912-20.

66. Mesko S, Sandler K, Cohen J, et al. Clinical outcomes for stereotactic ablative radiotherapy in oligometastatic and oligoprogressive gynecological malignancies. Int J Gynecol Cancer 2017;27:403-8.

67. Palma DA, Olson R, Harrow S, et al. Stereotactic ablative radiotherapy versus standard of care palliative treatment in patients with oligometastatic cancers (SABRCOMET): a randomised, phase 2, open-label trial. Lancet 2019;393:2051-8.

68. Iftode C, D’Agostino GR, Tozzi A, et al. Stereotactic body radiation therapy in oligometastatic ovarian cancer: a promising therapeutic approach. Int J Gynecol Cancer 2018;28:1507-13. 
Page 12 of 12

69. Lazzari R, Ronchi S, Gandini S, et al. Stereotactic body radiation therapy for oligometastatic ovarian cancer: a step toward a drug holiday. Int J Radiat Oncol Biol Phys 2018;101:650-60.

70. Macchia G, Lazzari R, Colombo N, et al. A large, multicenter, retrospective study on efficacy and safety of stereotactic body radiotherapy (SBRT) in oligometastatic ovarian cancer (MITO RT1 Study): a collaboration of MITO, AIRO GYN, and MaNGO groups. Oncologist 2020;25:e311-20.

71. Liu JF, Konstantinopoulos PA, Matulonis UA. PARP inhibitors in ovarian cancer: current status and future promise. Gynecol Oncol 2014;133:362-9.

72. Jiang X, Li W, Li X, et al. Current status and future prospects of PARP inhibitor clinical trials in ovarian cancer. Cancer Manag Res 2019;11:4371-90.

73. Reiss KA, Herman JM, Armstrong D, et al. A final report of a phase I study of veliparib (ABT-888) in combination with low-dose fractionated whole abdominal radiation therapy (LDFWAR) in patients with advanced solid malignancies and peritoneal carcinomatosis with a dose escalation in ovarian and fallopian tube cancers. Gynecol

Cite this article as: Flores-Balcázar CH, Urías-Arce DM. Radiotherapy in women with epithelial ovarian cancer: historical role, current advances, and indications. Chin Clin Oncol 2020;9(4):49. doi: 10.21037/cco-20-10
Flores-Balcázar and Urías-Arce. RT in women with epithelial OC

Oncol 2017;144:486-90.

74. Matz M, Coleman MP, Carreira H, et al. Worldwide comparison of ovarian cancer survival: histological group and stage at diagnosis (CONCORD-2). Gynecol Oncol 2017;144:396-404.

75. Hoppenot C, Eckert MA, Tienda SM, et al. Who are the long-term survivors of high grade serous ovarian cancer? Gynecol Oncol 2018;148:204-12.

76. Drake CG, Lipson EJ, Brahmer JR. Breathing new life into immunotherapy: review of melanoma, lung and kidney cancer. Nat Rev Clin Oncol 2014;11:24-37.

77. Coukos G, Tanyi J, Kandalaft LE. Opportunities in immunotherapy of ovarian cancer. Ann Oncol 2016;27:i11-15.

78. Herrera FG, Irving M, Kandalaft LE, et al. Rational combinations of immunotherapy with radiotherapy in ovarian cancer. Lancet Oncol 2019;20:e417-33.

79. Luke JJ, Lemons JM, Karrison TG, et al. Safety and clinical activity of pembrolizumab and multisite stereotactic body radiotherapy in patients with advanced solid tumors. J Clin Oncol 2018;36:1611-8. 